decrease in medical costs by $64 \%$, lost days by $69 \%$ and treatment delays by $77 \%$. Texas adopted EBTG in 2006; Total costs have declined by $50 \%$, patients are recovering more quickly, more providers are willing to treat these patients, opioid abuses have declined and many states are looking to adopt this model. Other state outcomes and essential elements needed in an EBTG will be discussed.

\section{DEVELOPING EVIDENCE-BASED CLINICAL PRACTICE GUIDELINES FOR PRIMARY CARE PHYSIOTHERAPY: A LOCAL INITIATIVE}

${ }^{1} S$ Bernhardsson, ${ }^{1,2} \mathrm{M}$ Larsson. ${ }^{1}$ Region Västra Götaland, Primary Care, Gothenburg, Sweden; ${ }^{2}$ Sahlgrenska Academy at Gothenburg University, Institute of Neuroscience and Physiology, Gothenburg, Sweden

\section{0:1136/bmjgs-2013-002293.81}

Background In primary care physiotherapy in Sweden, there is a lack of evidence-based clinical practice guidelines to support clinicians and patients in clinical decision making. A local initiative to develop and implement evidence-based clinical practice guidelines for PTs in primary care was initiated by a regional health authority.

Objectives To develop evidence-based clinical practice guidelines for physiotherapy treatment of low back pain, neck pain, and subacromial pain, for use in primary care physiotherapy in Sweden.

Methods The guideline development was performed by a project team of five primary care physiotherapists in a systematic process that was guided by a 7-step guideline development model by Grol et al. We performed systematic database searches, critical appraisal of the evidence base using GRADE, and formulated evidence-based practice recommendations.

Results The guideline format follows recommendations from AGREE II. The guidelines consist of a brief summary on the first page; a brief introduction to the topic with up-to-date information on definition of the condition, prevalence and prognosis; recommendations on patient management according to strength of evidence; a discussion section, and a detailed reference list. Detailed search strategy and search results, summaries of the body of evidence, recommended outcome measures, and patient information were provided in appendices to the guidelines.

Discussion Rigorous guideline development methodology was considered important to get clinicians' attention and approval - a requisite for regular use. Clinicians welcomed the initiative.

Implications for Guideline Developers It is important for clinician acceptance that guidelines are brief, to the point and relevant.

\section{CAN WE AUTOMATICALLY PRODUCE GENERIC DECISION AIDS FOR THE CLINICAL ENCOUNTER DIRECTLY FROM GRADE GUIDELINE RECOMMENDATIONS? EXPERIENCE FROM THE SHARE-IT PROJECT}

${ }^{1} \mathrm{~T}$ Agoritsas, ${ }^{2} \mathrm{~L}$ Brandt, ${ }^{2} \mathrm{~A}$ Anja Heen, ${ }^{2} \mathrm{~A}$ Kristiansen, ${ }^{3} \mathrm{P}$ Alonso-Coello, ${ }^{4} \mathrm{E}$ Akl, ${ }^{1} \mathrm{I}$ Neumann, ${ }^{1} \mathrm{~K}$ Tikkinen, ${ }^{5} \mathrm{~V}$ Montor, ${ }^{1} \mathrm{G}$ Guyatt, ${ }^{2} \mathrm{P}$ Vandvik. 'Department of Clinical Epidemiology and Biostatistics, McMaster University, Hamilton, Canada; ${ }^{2}$ Norwegian Knowledge Centre for the Health Services, Oslo, Norway; ${ }^{3}$ Iberoamerican Cochrane Center (CIBERESP), Hospital de Sant Pau, Barcelona, Spain; ${ }^{4}$ Department of Medicine, State University of New York City, Buffalo, USA; ${ }^{5}$ Knowledge and Evaluation Research Unit, Mayo Clinic, Rochester, MN, USA

10:1136/bmjqs-2013-002293.82
Background Although decision aids (DA) can help to communicate evidence to patients, their production is time consuming, often not based on the best available evidence or rapidly outdated. Linking trustworthy guidelines and DA for shared-decision making could both overcome these limitations and enhance guideline dissemination.

Objectives To test the feasibility of automatically translating any recommendations from GRADE guidelines into generic and interactive DA accessible on tablet computers for clinicians and their patients in the clinical encounter.

Methods As part of the DECIDE project, we developed a framework for translating components of GRADE into DA, following the International Patient Decision Aid Standards. Using a recently published guideline, we implemented that framework in our MAGIC (Making Grade the Irresistible Choice) application - a prototype electronic guideline tool and publication platform that can automatically display recommendations in multilayered presentation formats.

Results Our prototype was able to automatically translate a large number of GRADE recommendations and their supporting evidence into electronic and interactive DA. Preliminary results of user-testing in real patient-clinician interactions suggest that these DA can be used at the point of care to discuss estimates of treatment effects for patient relevant outcomes, confidence in estimates, burden of treatment, and cost issues.

Discussion This study provides a proof-of-concept that components of GRADE recommendations can be interactively displayed in generic tools for interactive shared-decision making in a wide range of treatment alternatives.

Implications for Guideline Developers/Users Our electronic DA offer promising opportunities to disseminate guidelines at the point of care.

\section{TOOLS FOR COMMUNICATING MAIN MESSAGES CONVEYED BY CLINICAL PRACTICE GUIDELINES (CPGS) - MAKING PATIENT RESOURCES USER FRIENDLY}

${ }^{1} \mathrm{C}$ Moga, ${ }^{2} \mathrm{D}$ Angus, ${ }^{1} \mathrm{D}$ Chojecki, ${ }^{1} \mathrm{C}$ Harstall, ${ }^{1 J} \mathrm{~J}$ Moffatt, ${ }^{3} \mathrm{P}$ Taenzer. ${ }^{1}$ Institute of Health Economics, Edmonton, Canada; ${ }^{2}$ Alberta Innovates - Health Solutions, Edmonton, Canada; ${ }^{3}$ University of Calgary, Faculty of Medicine, Calgary, Canada

\section{0:1136/bmjqs-2013-002293.83}

Background Background The Alberta Ambassador Guideline Adaptation Programme developed two CPGs on the management of low back pain and headache in primary care. Various guideline companion documents were developed for patients.

Objectives To review the process and outcomes of involving patients/public in the development of guideline resource documents.

Methods Focus groups of different compositions and using various sampling strategies were called upon: self-selected members from national patient pain association (patient information sheets); members of the public who serve on a standing Lay Committee (patient information sheets, comic book); purposive recruitment by market research company (comic book). Participants answered questions related to the content, format, and presentation of patient resources using different interview methods.

Results Discussion themes included wordiness of documents, language accessibility, use of logos, and trustworthiness. Multiple suggestions for improvements that were sensitive to the needs of participants were considered before publication of final products. The comic book was not published. 
Discussion Even though the focus group procedures varied, there was significant overlap and repetition in the feedback received on the same guideline resources. The patient focus group facilitated by a clinician engaged participants in discussions oriented to clinical issues. The comic book was considered to be a novel communication vehicle by clinicians but not so by public.

Implications for Guideline Developers/Users Involving a motivated Lay Committee facilitated by someone not directly related to the project seems to be a valuable alternative to other focus groups of patients which may require more effort and resources.

\section{IMPLEMENTING NUTRITION GUIDELINES THROUGH PRACTICE TOOLS FOR REGISTERED DIETITIANS}

K Kren, L Moloney, P Ziegler, A Acosta. Academy of Nutrition and Dietetics, Chicago, USA

\section{0:1136/bmjqs-2013-002293.84}

Background Evidence Based Dietetics Practice Toolkits are resources intended to assist registered dietitians (RDs) in implementing nutrition guidelines derived from systematic reviews.

Objectives Surveys, sent at least one year past publication, were used to explore who uses toolkits, how toolkits are used, and if RDs find toolkits useful for implementation of guidelines.

Methods A standard questionnaire was sent to 1379 individuals for six toolkits (Celiac Disease, Critical Illness, Diabetes, Heart Failure, Oncology, and Paediatric Overweight) in 2011 and 2012, using Survey Monkey.

Results Responses received were 131 (9\%), of which 42\% were RDs in practice $>15$ years, in direct care (63\%), and in settings of outpatient (51\%) and inpatient (31\%). Respondents mostly used toolkits for patient care (66\%), nutrition counselling (40\%) and development of forms (37\%). Regarding the tools provided, $66 \%$ of respondents indicated that they found the summary of nutrition care and patient education materials to be very/somewhat useful or useful. The same was true for $63 \%$ for the case studies, $61 \%$ for the flowchart of patient encounters, $60 \%$ for the patient encounter process instructions and $56 \%$ for the sample documentation forms. Some respondents (24\%) seldom or never used the guidelines prior to toolkit use; however, 65\% indicated that the toolkit was useful in translating the guidelines to practice.

Discussion The implementation of guidelines is often overlooked yet is crucial to changing practice. These toolkits allow a useful strategy for assisting with implementation. Similar practice tools, tailored to practitioners and setting, may be useful in guideline implementation for a variety of conditions.

\section{TRANSFORMING EVIDENCE FROM MULTIPLE GUIDELINES INTO USER FRIENDLY CLINICAL PRACTICE TOOLS FOR REMOTE CANCER TREATMENT-RELATED SYMPTOM MANAGEMENT: THE COSTARS PROJECT}

${ }^{1,2} \mathrm{D}$ Stacey, ${ }^{2,3} \mathrm{M}$ Carley, ${ }^{3} \mathrm{M}$ Harrison, ${ }^{4}$ Pan-Canadian Oncology Symptom Triage and Remote Support (COSTaRS), Group. 'University of Ottawa, Ottawa, Canada; ${ }^{2}$ Ottawa Hospital Research Institute, Ottawa, Canada; ${ }^{3}$ Queen's University, Kingston, Canada; ${ }^{4}$ Canada

\section{0:1136/bmjqs-2013-002293.85}

Objectives To transform evidence from guidelines for clinical practice tools for remote management of patients experiencing symptoms related to cancer treatment. Clinical practice protocols are defined as user-friendly knowledge translation tools to support patient care. These tools narrow the know-do gap by presenting the best available evidence from guidelines while using a format that is sensitive to how nurses think and what nurses do. Methods Mixed methods descriptive study guided by CANIMPLEMENT $\odot$. The process involved: a) conducting a systematic search for guidelines; b) developing symptom-specific protocols using evidence from quality appraised clinical practice guidelines; c) reaching consensus on the clinical practice protocol template, and d) validating the clinical practice protocols.

Results Clinical practice protocols were developed and validated for 13 symptoms using 42 clinical practice guidelines with a median of 3 guidelines per protocol (range 1 for bleeding to 7 vomiting). For the first two protocols, source guideline AGREE rigour subscale ratings ranged from $8 \%$ to $86 \%$ (median 60.1; diarrhoea; 40.5 fever). The protocols were developed using guidelines, symptom severity questions included the Edmonton Symptom Assessment System, and iterative feedback from practicing nurses. Usability testing revealed: high readability, just the right amount of information, and appropriate terms. Access to protocols needs to be tailored to individual practices (e.g. electronic application, access to paper-based versions). Nurses requested training and support to implement them.

Discussion These tools, created from guidelines, transform evidence into user-friendly protocols for use by nurses when guiding patients at home to better manage their cancer treatmentrelated symptoms.

\section{USE OF NON-RANDOMISED STUDIES IN THE GUIDELINE PROCESS: THE GRADE APPROACH}

N Santesso, R Mustafa, H Schunemann, C4-GEP Methodology Group. McMaster University, Hamilton, Canada

\section{0:1136/bmjqs-2013-002293.86}

Background The GRADE approach to guideline development requires a review of the best available evidence which includes randomised controlled trials (RCTs) and non-randomised studies (NRS).

Objectives Describe the use of NRS as a replacement, a sequence, or a complement for RCTs, in a World Health Organization guideline using the GRADE approach.

Methods We searched the literature using no study type limits for the effect of screening and treatment of precancerous lesions on patient or population important outcomes and for baseline risks. We assessed quality of the evidence using GRADE.

Results Depending on the outcomes, we found few to no RCTs. When there was low/very low overall quality evidence from RCTs, we used NRS studies with no independent control groups to compare proportions between groups and calculate a relative effect of treatment and this evidence replaced the RCT evidence with similar/higher quality evidence. We found no evidence in RCTs for long-term outcomes, such as spontaneous abortion. Therefore, we used data from NRS (cohort studies) for premature delivery (a surrogate) to provide sequential evidence. For evidence about baseline risk of precancerous lesions and other outcomes, we used NRS a complement to the RCT data.

Discussion Data from NRS provided evidence in three ways. One key criterion to consider when grading this evidence is indirectness due to indirect comparisons, surrogate outcomes or varying population risks. 